\title{
Reduction of Cardiac Cell Death after Helium Postconditioning in Rats: Transcriptional Analysis of Cell Death and Survival Pathways
}

\author{
Gezina TML Oei, ${ }^{1}$ Michal Heger, ${ }^{2}$ Rowan F van Golen, ${ }^{2}$ Lindy K Alles, ${ }^{2}$ Moritz Flick, ${ }^{1}$ \\ Allard C van der Wal, ${ }^{3}$ Thomas M van Gulik, ${ }^{2}$ Markus W Hollmann, ${ }^{1}$ Benedikt Preckel, ${ }^{1}$ and Nina C Weber ${ }^{1}$ \\ ${ }^{1}$ Department of Anesthesiology, Laboratory of Experimental Intensive Care and Anesthesiology (L.E.I.C.A.), ${ }^{2}$ Department of \\ Experimental Surgery, and ${ }^{3}$ Department of Pathology, Academic Medical Center, University of Amsterdam, Amsterdam, The \\ Netherlands
}

\begin{abstract}
Helium, a noble gas, has been used safely in humans. In animal models of regional myocardial ischemia/reperfusion (I/R) it was shown that helium conditioning reduces infarct size. Currently, it is not known how helium exerts its cytoprotective effects and which cell death/survival pathways are affected. The objective of this study, therefore, was to investigate the cell protective effects of helium postconditioning by PCR array analysis of genes involved in necrosis, apoptosis and autophagy. Male rats were subjected to $25 \mathrm{~min}$ of ischemia and 5, 15 or $30 \mathrm{~min}$ of reperfusion. Semiquantitative histological analysis revealed that $15 \mathrm{~min}$ of helium postconditioning reduced the extent of I/R-induced cell damage. This effect was not observed after 5 and 30 min of helium postconditioning. Analysis of the differential expression of genes showed that 15 min of helium postconditioning mainly caused upregulation of genes involved in autophagy and inhibition of apoptosis versus I/R alone. The results suggest that the cytoprotective effects of helium inhalation may be caused by a switch from pro-cell-death signaling to activation of cell survival mechanisms, which appears to affect a wide range of pathways.
\end{abstract}

Online address: http://www.molmed.org

doi: $10.2119 / \mathrm{molmed} .2014 .00057$

\section{INTRODUCTION}

Coronary artery disease and subsequent myocardial infarction is a common cause of death (1). In the acute phase of myocardial infarction, occlusion of a cardiac vessel by a thrombus or stationary embolus leads to myocardial hypoxia, which is followed by cessation of aerobic respiration and ATP production in the affected cardiomyocytes. The rapid energy depletion gradually suppresses metabolic activity and leads to the induction of cell death pathways and eventually the demise of cardiomyocytes. Reperfusion and reoxygenation of the infarcted tissue, as a result of, for example, pharmacological dissolution or dislodgement of the clot, ameliorate the extent of hypoxia-induced cell death, but in turn inflict lethal reperfusion injury (2). The type of cell death that is manifested depends on how fast reoxygenation occurs as a result of reperfusion (2) and may proceed via necrosis, apoptosis or autophagy. Cell survival is mediated mainly by activation of antiapoptotic

Address correspondence to Markus W Hollmann, Department of Anesthesiology, Academic Medical Center, University of Amsterdam, Meibergdreef 9, 1105 AZ Amsterdam, The Netherlands. Phone: +31-(0)20-566-3630 or +31-(0)20-566-3633 (secretary); Fax: +31-(0)20-6979441;

E-mail:m.w.hollmann@amc.uva.nl.

Submitted March 25, 2014; Accepted for publication August 26, 2014; Epub

(www.molmed.org) ahead of print August 26, 2014.

\section{The Feinstein Institute for Medical Research Empowering Imagination. Pioneering Discovery.}

proteins and stimulation of prosurvival autophagy (3-5).

The use of the colorless, odorless, nonanesthetic noble gas helium in patients with respiratory disease was first described in 1934 (6). The benefits of helium mixed with oxygen (heliox) were attributed to its reduced density and consequently reduced work of breathing in respiratory conditions. In the last few years, the use of heliox has been tested in acute respiratory diseases in both children and adults $(7,8)$ and for decompression illness (9). In addition, helium has been shown to reduce the extent of cell death in myocardial, neuronal and epithelial tissue subjected to ischemia/reperfusion (I/R), as reviewed in (10). In the heart, helium preconditioning considerably reduces infarct size in rat and rabbit models of cardiac I/R by coronary artery ligation (data of these studies are summarized in Supplementary Figure S1). On average, the infarct size reduction was $18 \%$ to $25 \%$ after exposure to helium preconditioning 
(11-20). Helium postconditioning (HePOC), the clinically more relevant form of conditioning, also protects the myocardium, as has been demonstrated in several rat strains $(15,21)$. Some mechanistic insight into the damage-ameliorating effects of helium gas during I/R has been provided in the last few years (22). Apoptotic pathways have been shown to be involved in helium conditioning (17-19).

Given the fact that helium preconditioning and HePOC reduce the extent of I/R-induced cell death, helium may affect the transcriptional regulation of cell death and survival pathways and thereby promote prosurvival signaling. Here we used a regional cardiac $\mathrm{I} / \mathrm{R}$ model in rats to determine the differential expression patterns of genes related to apoptosis, necrosis and autophagy following ischemia, I/R or I/R with different regimes of HePOC. Transcriptional analysis of these pathways not only allowed us to test the hypothesis that HePOC reduces the magnitude of death signaling and stimulates survival pathways, but also provided insight into the significance of each mode of cell death and cell survival in every phase of I/R under native conditions and following HePOC.

\section{MATERIALS AND METHODS}

\section{Animal Model of Ischemia/Reperfusion}

Animal experiments were approved by the Academic Medical Center's animal ethics committee (DAA102650). Animals were treated in accordance with the Guide for the Care and Use of Laboratory Animals (1996) (24). Male Wistar rats (354 to $426 \mathrm{~g}$, age range of 12 to $16 \mathrm{wks}$ ) were acclimatized for $7 \mathrm{~d}$ under conditions of 12-h light and dark cycles and ad libitum access to food and water.

Rats were anesthetized and surgically prepared as described previously (21). In short, rats were mechanically ventilated and cannulation of the carotid artery was done for measurement of the mean arterial pressure and heart rate and for blood sampling. The left anterior descending coronary artery (LAD) was lig-

A Histological analysis

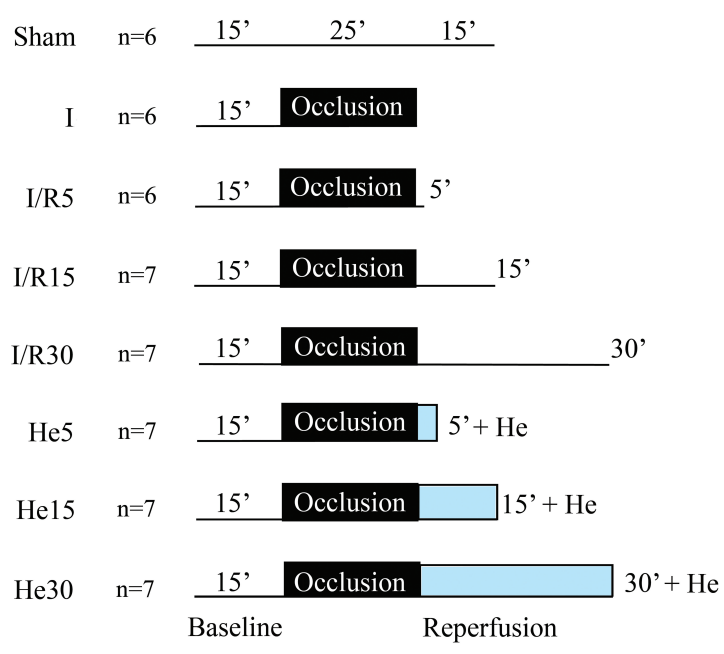

B qRT-PCR

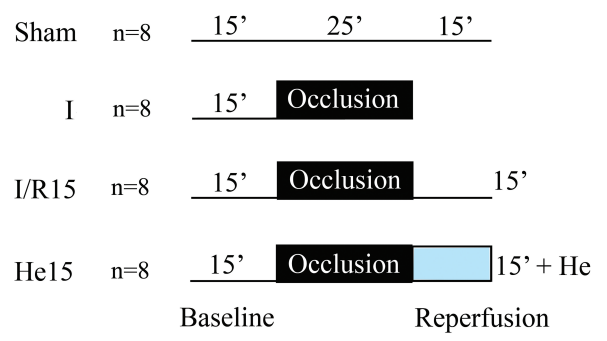

Figure 1. Schematic overview of the experimental protocols. (A) Experimental setup for histological analysis. Rats were euthanized after $25 \mathrm{~min}\left(25^{\prime}\right)$ of ischemia and 5, 15, or $30 \mathrm{~min}$ $\left(5^{\prime}, 10^{\prime}\right.$, or 30') of reperfusion (groups I/R5, I/R15, I/R30; the group I had ischemia alone; the sham group had no I/R. The number behind the reperfusion block indicates the duration (in minutes) of the reperfusion phase. Helium postconditioning $(\mathrm{He}$ ) encompassed the entire reperfusion phase (groups $\mathrm{He} 5, \mathrm{He} 15, \mathrm{He} 30$ ). (B) Experimental setup for gene analysis by QRT-PCR. Rats were euthanized after $25 \mathrm{~min}$ of ischemia or after $15 \mathrm{~min}$ of reperfusion.

ated with a single puncture 5-0 Prolene suture (Ethicon [Johnson\&Johnson], Amersfoort, the Netherlands) through the myocardium. The ends of the suture were threaded through propylene tubing to enable tightening and loosening of the snare for the induction of I/R. The helium postconditioning groups received helium gas (Linde Gas Benelux, Dieren, the Netherlands) at the onset of reperfusion (Figure 1). To make sure that sufficient helium was present in the lungs and normal air washed out at the onset of reperfusion, helium administration was started at $24 \mathrm{~min}$ of ischemia.

\section{Study Design (Figure 1)}

The study was divided into two test arms for the determination of (1) histological damage, and (2) quantitative reverse transcriptase polymerase chain reaction (qRT-PCR) experiments. Additionally, Western blot experiments were performed to investigate levels of proteins involved in autophagy in the I/R15 and He15 groups (I/R15 received 15 min of reperfu- 
sion; He15 received 15 min of helium postconditioning; for additional information on the groups, see Figure 1 legend).

\section{Histological Staining and Analysis}

For histological processing of the hearts, the organs were mounted on a modified Langendorff setup and perfused retrograde with isotonic saline solution to wash out blood from the coronary circulation. Next, the coronary circulation was flushed with $10 \mathrm{~mL}$ of icecold fixative (96\% ethanol, acetic acid, $10 \%$ buffered formalin and Milli-Q water [Millipore, Amsterdam, the Netherlands] in a 50:5:10:35 volume ratio). Adequate retrograde perfusion was confirmed visually by uniform changes in size and color of the myocardium. The heart was then removed from the Langendorff setup and stored in ice-cold fixative. Fixed rat hearts were dehydrated in graded steps of ethanol and xylene and cut in half, longitudinally at the center of the ligation of the LAD (Supplementary Figure S2). The left half of the heart was embedded in paraffin and sectioned with a microtome along the cutting plane (4- $\mu \mathrm{m}$ thick sections). The sections were deparaffinized, stained with hematoxylin and eosin (H\&E) and mounted using VectaMount (Vector Laboratories, Burlingame, CA, USA).

A histological scoring system was developed to semiquantitatively analyze the extent of myocardial damage in I/Rsubjected rat hearts and to determine the extent of cardiomyocyte protection by HePOC. The scoring parameters are listed in Table 1. H\&E-stained sections were viewed under a light microscope (confocal microscope SP8 X; Leica, Rijswijk, the Netherlands). The area at risk was delineated on the basis of the pathological demarcation zone at low power view (10x magnification), whereby the puncture wound from the suture was used as an anchor point. The area at risk always extended from the periphery of the puncture wound to the apical end. Semiquantitative scoring of histopathological parameters of myocardial damage (contraction band necrosis, interstitial edema, granulocyte adherence/extravasation, ex-

Table 1. Histological scoring parameters used to semi-quantify myocardial slices.

\begin{tabular}{lcl}
\hline Parameter & Score & Quantitative value \\
\hline 1. Contraction bands/coagulation necrosis & 0 & Absent \\
& 1 & $1 \%-10 \%$ of cardiomyocytes \\
2. Interstitial edema & 2 & $11 \%-50 \%$ of cardiomyocytes \\
3. Granulocyte infiltration & 3 & $51 \%-100 \%$ of cardiomyocytes \\
4. Platelet aggregates/thrombi & 0 & Absent \\
5. Extravasation of red blood cells & 1 & Present \\
& 0 & Absent \\
& 1 & Present \\
\end{tabular}

travasation of erythrocytes) was performed at higher power magnification $(400 \times)$ in the full thickness of the myocardium. The endocardial segment and an epidcardial segment of myocardium were scored separately. All parameters were scored in four fields of view (FOVs) per segment in the area at risk (400×).

\section{qRT-PCR}

At the end of the experiment (Figure 1B), the heart was excised under deep anesthesia. The area at risk (AAR) was cut from the rest of the myocardium (area not at risk [NAAR]) and sliced in two pieces on ice, which was snap frozen in liquid nitrogen and stored at $-80^{\circ} \mathrm{C}$ until further analysis. All analyses were performed in myocardial tissue at risk. For a detailed description of RNA extraction, cDNA synthesis and run parameters, see Supplementary Table S1.

Melting curve analysis was performed at the end of the PCR run. Amplicons that showed amplification of nonspecific products were excluded from analysis. The data was further processed according to Ruijter and colleagues (24). Each amplicon was corrected for baseline fluorescence and a common fluorescence threshold $\mathrm{Nq}$ for all arrays was set in the upper half of the log-linear phase of the amplification plot. Amplicons that did not reach $\mathrm{Nq}$ before cycle 40 were considered undetectable. Next, the individual PCR efficiencies were calculated and amplicons with an efficiency $<1.80$ or $>2.00$ were excluded from further analysis. The individual effi- ciencies were subsequently used to calculate the starting concentration $\left(\mathrm{N}_{0}\right)$ per amplicon. All samples were normalized to the mean $\mathrm{N}_{0}$ of the housekeeping genes that showed the most stable expression over all arrays (that is, Ldha and Rplp1).

Two comparisons were made. First, the differences between experimental groups (ischemia, I/R15 and He15) and the sham group was calculated according to

$\frac{\frac{\mathrm{N}_{0} \mathrm{GOI}}{\mathrm{N}_{0} \text { Housekeeping }} \mathrm{I} / \mathrm{R}}{\frac{\mathrm{N}_{0} \mathrm{GOI}}{\mathrm{N}_{0} \text { Housekeeping }} \text { sham }}$

and expressed as fold difference versus the sham group. The GOI (gene of interest) within one group is thus first normalized against the housekeeping gene, afterward a comparison between each experimental group and the sham group was made. Heat maps of these expression profiles were generated using Mayday Microarray Data Analysis software (25). A total of 84 genes involved in cell death and survival pathways were investigated and divided in four categories: necrosis, proapoptosis, antiapoptosis and autophagy. For a description of each gene also see Supplementary Table S2. Due to multiple roles for some genes, the total number of genes in each category was 27 (necrosis), 23 (proapoptosis), 14 (antiapoptosis) and 33 (autophagy). After exclusion of genes with insufficient n-numbers in either group due to melting curve, efficiency or undetectable levels of mRNA, a total of 
20 (necrosis), 21 (proapoptosis), 14 (antiapoptosis) and 29 (autophagy) genes were included in the heat maps.

Secondly, a comparison between the I/R and He15 group was made. For this, the means of the He15 group were divided by the means of the I/R group and presented as fold increase. Gene selection was performed according to the criteria as described above. A total of 20 (necrosis), 20 (proapoptosis), 14 (antiapoptosis) and 30 genes (autophagy) were included in the analysis.

\section{Western Blot Analysis}

Western blot analysis was performed in AAR and NAAR tissue to allow for investigation of the two distinct types of tissue within one sample. For preparation of cytosol, membrane and mitochondrial fractions as well as for Western blot analysis, see "Methods: Western Blot Analysis" in the Supplement Data. In short, samples were loaded on a Criterion Gel. Proteins were separated by electrophoresis and transferred onto an Immobilin-FL membrane. The membrane was incubated overnight at $4^{\circ} \mathrm{C}$ with beclin1 (Cell Signaling Technology, Leiden, the Netherlands, 1:1000) or sequestosome1 (Abcam, Cambridge, MA, USA, 1:2000) antibody. After washing in fresh, cold TBS-T, the blot was subjected to the appropriate horseradish peroxidase conjugated secondary antibody for $1 \mathrm{~h}$ at room temperature. Subsequently, immunoreactive bands were visualized on an Odyssey Infrared Imaging System. Densitometric analysis of the blots was performed in Odyssey Infrared Scanning Software. Results are presented as the ratio of the target protein over the fraction-specific control protein: for the cytosol fraction this was actin (1:5000), for the membrane fraction NaKATPase (Cell Signaling Technology, 1:5000), for the mitochondrial fraction PHB1 (Cell Signaling Technology, 1:10000).

\section{Statistical Analysis}

Statistical analysis was performed in GraphPad Prism (GraphPad Software, La Jolla, CA, USA). Baseline hemodynamics were analyzed using one way analysis of

Table 2. Hemodynamics (mean arterial pressure and heart rate) sampled after 15 min of baseline, 24 min of ischemia and 15 min of reperfusion. ${ }^{a}$

\begin{tabular}{|c|c|c|c|c|c|}
\hline Group $^{b}$ & Baseline & $\begin{array}{l}\text { After } 24 \mathrm{~min} \\
\text { of ischemia }\end{array}$ & $\begin{array}{l}\text { After } 5 \text { min } \\
\text { of reperfusion }\end{array}$ & $\begin{array}{l}\text { After } 15 \text { min } \\
\text { of reperfusion }\end{array}$ & $\begin{array}{l}\text { After } 30 \text { min } \\
\text { of reperfusion }\end{array}$ \\
\hline \multicolumn{6}{|c|}{ Mean $\mathrm{AP}^{\mathrm{c}}(\mathrm{mmHg})$} \\
\hline Sham & $111 \pm 13$ & $113 \pm 22$ & $113 \pm 21$ & $98 \pm 20$ & \\
\hline I & $95 \pm 16$ & $110 \pm 15$ & & & \\
\hline I/R5 & $90 \pm 33$ & $95 \pm 19$ & $73 \pm 20$ & & \\
\hline I/R15 & $95 \pm 24$ & $93 \pm 20$ & $83 \pm 21$ & $62 \pm 8$ & \\
\hline I/R30 & $96 \pm 29$ & $91 \pm 28$ & $80 \pm 24$ & $79 \pm 21$ & $60 \pm 15$ \\
\hline $\mathrm{He} 5$ & $102 \pm 20$ & $108 \pm 23$ & $78 \pm 21$ & & \\
\hline $\mathrm{He} 15$ & $102 \pm 20$ & $108 \pm 17$ & $96 \pm 14$ & $75 \pm 11$ & \\
\hline $\mathrm{He} 30$ & $97 \pm 25$ & $100 \pm 24$ & $95 \pm 18$ & $91 \pm 23$ & $69 \pm 15$ \\
\hline \multicolumn{6}{|c|}{ Mean $\mathrm{HR}^{\mathrm{d}}(\mathrm{BPM})$} \\
\hline Sham & $372 \pm 20$ & $373 \pm 20$ & $363 \pm 24$ & $352 \pm 32$ & \\
\hline I & $341 \pm 40$ & $367 \pm 32$ & & & \\
\hline I/R5 & $338 \pm 29$ & $354 \pm 29$ & $330 \pm 18$ & & \\
\hline I/R15 & $320 \pm 60$ & $340 \pm 50$ & $326 \pm 46$ & $306 \pm 44$ & $320 \pm 39$ \\
\hline I/R30 & $329 \pm 62$ & $359 \pm 39$ & $342 \pm 38$ & $346 \pm 41$ & \\
\hline He5 & $360 \pm 36$ & $366 \pm 16$ & $344 \pm 35$ & & \\
\hline Hel5 & $338 \pm 35$ & $365 \pm 36$ & $350 \pm 37$ & $324 \pm 34$ & \\
\hline He30 & $343 \pm 57$ & $366 \pm 36$ & $347 \pm 47$ & $359 \pm 40$ & $328 \pm 51$ \\
\hline
\end{tabular}

${ }^{a}$ Data are shown as mean $\pm S D$. At each time point there were no significant differences between different experimental groups.

${ }^{b}$ On group infomation, see Materials and Methods and Figure 1.

${ }^{c}$ Mean AP, mean arterial pressure in $\mathrm{mmHg}$.

${ }^{\mathrm{d}}$ Mean HR, mean heart rate in beats/minute.

variance (ANOVA) with a Tukey post hoc test for multiple comparisons. Differences in mRNA expression and protein levels between the I/R and He15 group were tested using a Mann-Whitney test. A $P$ value of $<0.05$, indicated with an asterisk in the figures, was considered significant.

All supplementary materials are available online at www.molmed.org.

\section{RESULTS}

\section{Hemodynamic Parameters}

Aortic pressure and heart rate (mean \pm $\mathrm{SD})$ during the experiments are shown in Table 2. Baseline hemodynamics did not vary between groups.

\section{Histological Damage Profiles}

Histological analysis was performed to assess cell damage at a microscopic level ( $n=4-6$ per group). The morphology of cardiomyocytes clearly differed among the experimental groups. Hearts in the sham group contained normal unaltered cardiomyocytes; myofibrils were ordered in a structured manner and exhibited similar morphology. The absence of cardiomyocyte damage, inflammatory cell infiltrates, red blood cell extravasation, thrombosis and edema resulted in a mean total histology scores of 0 . Representative micrographs of sham-operated animals are shown in Figure 2B and Supplementary Figure S3.

After 25 min of ischemia, hypercontraction of myofibers was observed in some slices (Supplementary Figure S4), without loss of the native structure and morphology of cardiomyocytes similar to the sham-operated group. In the 5-min reperfusion group, waviness of myofibers and contraction bands could be observed and were accompanied by other signs of tissue damage (Supplementary Figure S5). At 15 or 30 min of reperfusion, the cardiac tissue exhibited clear signs of damage that entailed necrosis, interstitial edema, granulocyte 
A

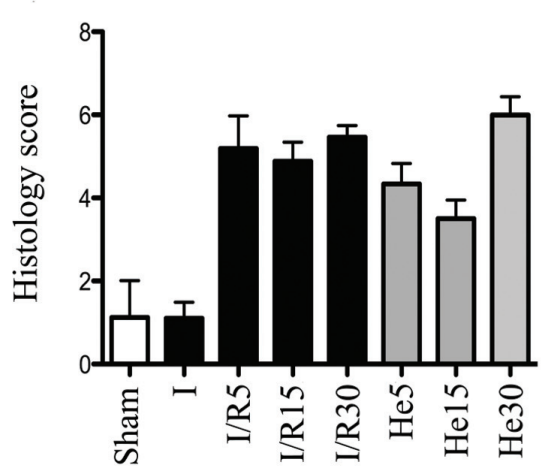

$\mathrm{C}$

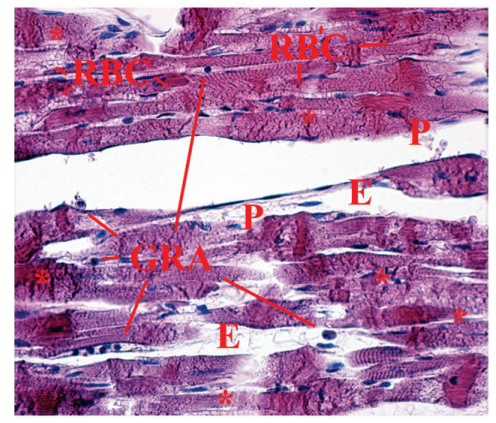

B

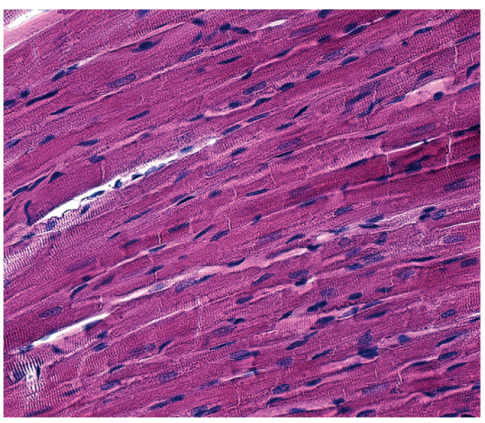

$\mathrm{D}$

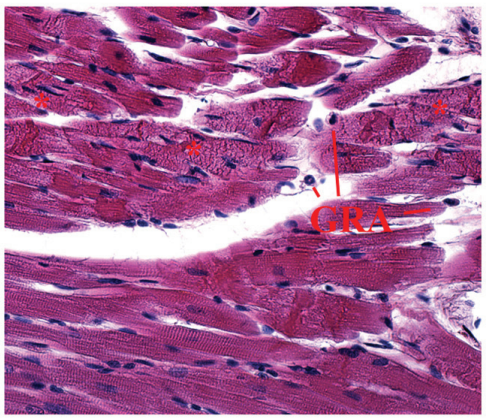

Figure 2. (A) Total histology score plotted per group. Representative histological sections of (B) hearts in the sham, (C) I/R15, and (D) He15 group. E indicates tissue edema; P, presence of platelets and thrombi; RBC, extravasation of red blood cells; GRA, extravasation of granulocytes; asterisks $\left(^{*}\right)$, contraction band or coagulation necrosis. Original magnification 400x.

infiltration, platelet aggregates/thrombi and extravasation of red blood cells (Figure 2C). This was observed in all histological specimens (Supplementary Figures S6, S7) and was reflected in the total histology score (Figure 2A).

Fifteen min of HePOC reduced the extent of cell damage, which was reflected in the trend of an overall lower total histology score in this group compared with all other intervention groups (Figure 2A). Interestingly, 15 min of HePOC (Supplementary Figure S9) resulted in less cell damage compared with 30 min of helium (Supplementary Figure S10), indicating that prolonged helium inhalation is not beneficial for cardiac I/R. The morphological features of cardiomyocytes that had been exposed to $30 \mathrm{~min}$ of HePOC were similar to those of cardiomyocytes in the I/R15, I/R30 and He5 group (Supplementary Figures S6, S7, S8). A representa- tive micrograph of myocardium exposed to I/R15 and He15 is shown in Figures 2C and $\mathrm{D}$, respectively. The extent of necrosis, edema, extravasation of red blood cells, granulocyte infiltration and platelet aggregation is much lower after He15 (see Figure 2D) compared with I/R15 (see Figure $2 \mathrm{C}$ ). This is also reflected in the total histology score (see Figure 2A).

\section{mRNA Expression Profiles in Hearts Subjected to Ischemia, Ischemia/Reperfusion and HePOC}

Exposure of cardiomyocytes to ischemia, I/R15 and He15 caused differential gene expression in all cell death pathways compared with cardiomyocytes in the sham-operated group (Figure 3). Many genes of the cell death pathways in the ischemia and I/R15 groups are regulated in a similar manner, that is, the expression patterns of genes involved in the execu- tion of necrosis, apoptosis and autophagy are affected similarly under both conditions of ischemia and I/R. To specify this: most genes are similarly up- or downregulated after ischemia and I/R15. This can be seen from the actual number of genes that are up- or downregulated in each group of genes. Fifteen of 20 genes involved in necrosis pathways were downregulated after ischemia and 14 of 20 after I/R15. Simultaneously, genes involved in proapoptosis were upregulated (I, 11 of 21; I/R15, 12 of 21) and antiapoptotic genes were downregulated (10 of 14 in both groups). Ischemia and I/R15 also downregulated most genes involved in autophagy: 21 of 29 and 23 of 29 , respectively.

The addition of $15 \mathrm{~min}$ of helium postconditioning changed the expression profiles of whole sets of genes. This can be seen easily when one compares the last column to the third column. Helium particularly upregulated genes involved in antiapoptotic pathways (10 of 14) and autophagy (16 of 29). By contrast, ischemia and I/R15 downregulated the majority of antiapoptotic genes and genes involved in autophagy. This suggests that the protective effect of $15 \mathrm{~min}$ of helium postconditioning is linked to expression of genes involved in autophagy and antiapoptotic signaling.

Figure 3 first of all shows that gene expression patterns that are visible during reperfusion already emerge during ischemia. Additionally, it shows that exposure to a short 15-min episode of HePOC is powerful enough to exert changes on expression patterns of genes involved in cell death pathways, which occurs already during early reperfusion. Taken together, the data suggests that the effects of helium postconditioning are immediate and undo some of the detrimental changes in gene expression that have been initiated during ischemia and are extended during reperfusion.

\section{Effects of HePOC on Cell Death, Cell Survival and Autophagy}

Beside the general changes in gene expression profiles during ischemia, reperfusion and POC in comparison to sham 


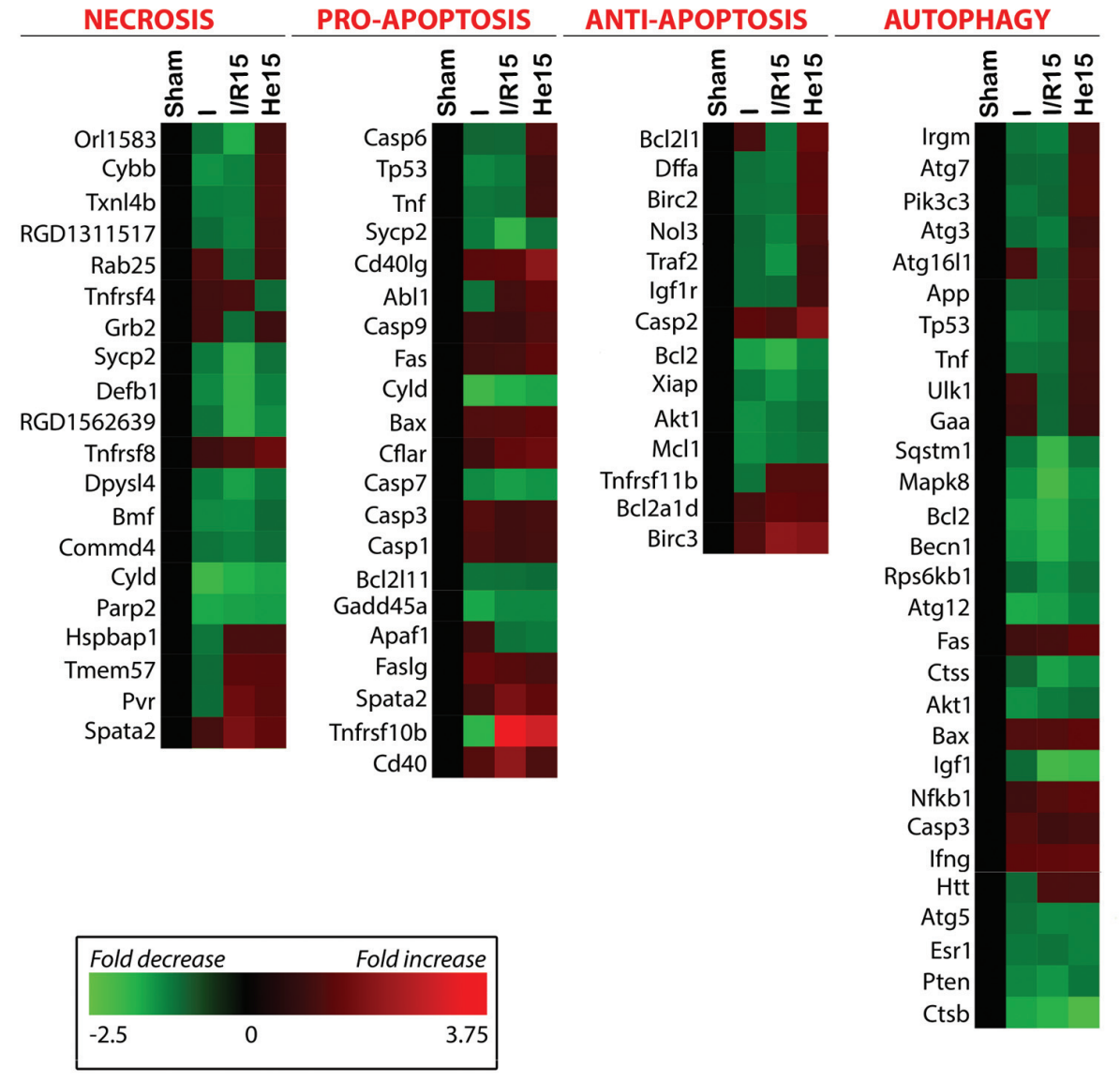

Figure 3. Heat maps indicating the differential expression of genes in the ischemia $(I), I / R$, and I/R + He (He15) relative to the sham group, classified per cell death/survival pathway and autophagy. The significance of the color-coding, indicating the extent of fold decrease (green) and fold increase (red), is provided in the inserted legend. The function(s) of the genes is (are) summarized in Supplementary Table S2.

animals, a direct comparison between the I/R15 and He15 group has been made. In Figure 4, the fold increase and decrease of each gene within one of four categories of cell damage/survival after He15 in comparison to I/R15 is depicted. This figure shows which genes are upregulated and downregulated (most strong upregulation to downregulation from left to right in the figure) after HePOC and could therefore play a key role in helium's underlying cardioprotective mechanism.

In line with results shown in Figure 3, in comparison to I/R15, He15 particularly upregulates genes involved in autophagy and antiapoptosis: 27 of 30 genes involved in autophagy were upregulated, and 12 of 14 antiapoptotic genes. This underlines the general finding that the cardioprotective mechanism of HePOC is related to an increase in the expression of genes employed in autophagy and against apoptosis.

Apart from general trends, individual genes were found to be significantly upregulated after He15 as compared to I/R15. Within the necrosis group of genes, Olr1583, Sycp2, Cybb, Txnl4b and Dpysl4 all were significantly upregulated after He15 as compared to I/R15 only. Their function in vivo is described in Supplementary Table S2 and the relation of these genes to HePOC will be addressed in the discussion.

\section{Protein Levels of Beclin-1 and Sequestosome}

Differences in protein levels of beclin-1 and sequestosome between the I/R15 and
He15 groups were consistent in AAR and NAAR tissue, indicating a tissueindependent effect of helium inhalation. In the mitochondrial fraction, $15 \mathrm{~min}$ of helium postconditioning significantly increased the amount of beclin-1, which was found in both the AAR and NAAR tissue (see also Figure 5). No differences in sequestosome protein levels could be found between the I/R15 and He15 groups. For an overview of sequestosome Western blot results, see Supplementary Figure S11.

\section{DISCUSSION}

In this study, helium-induced postconditioning was investigated by microscopic assessment of cell damage and analysis of its transcriptional effects on cell death and survival pathways (necrosis, apoptosis and autophagy). We showed that signs of cell damage in H\&E-stained histological slices were reduced after $15 \mathrm{~min}$ of helium. Additionally we showed that in comparison to I/R15 only, He15 upregulated genes in all categories; necrosis, proand antiapoptosis and autophagy. However, He15 predominantly upregulated genes involved in autophagy and inhibition of apoptosis. Taken together, these data suggested that the HePOC-induced reduction of I/R-induced cell damage is mediated by an instantaneous upregulation of genes employed in autophagy and the inhibition of apoptosis during early reperfusion. We therefore suggest that the upregulation of these genes at least counterbalances or even overrules the upregulation of the pro-cell-death genes, resulting in cardioprotection after HePOC.

In earlier studies from our laboratory, infarct size reduction (analyzed by triphenyl tetrazolium chloride [TTC]-Evans blue staining) after HePOC was found in several rat strains $(15,21,26)$. Fifteen minutes of $70 \%$ helium during early reperfusion reduced infarct size as a percentage of area at risk from $47 \% \pm 2 \%$ (mean \pm SEM) in control to $30 \% \pm 2 \%$ in the HePOC group (Supplementary Figure S12), while the area at risk as percentage of total ventricular tissue was similar in both groups; $21 \% \pm 2 \%$ in $\mathrm{CON}$ and $22 \% \pm 2 \%$ (data not shown). This is in line with the results of 


\section{He15 vs I/R15}
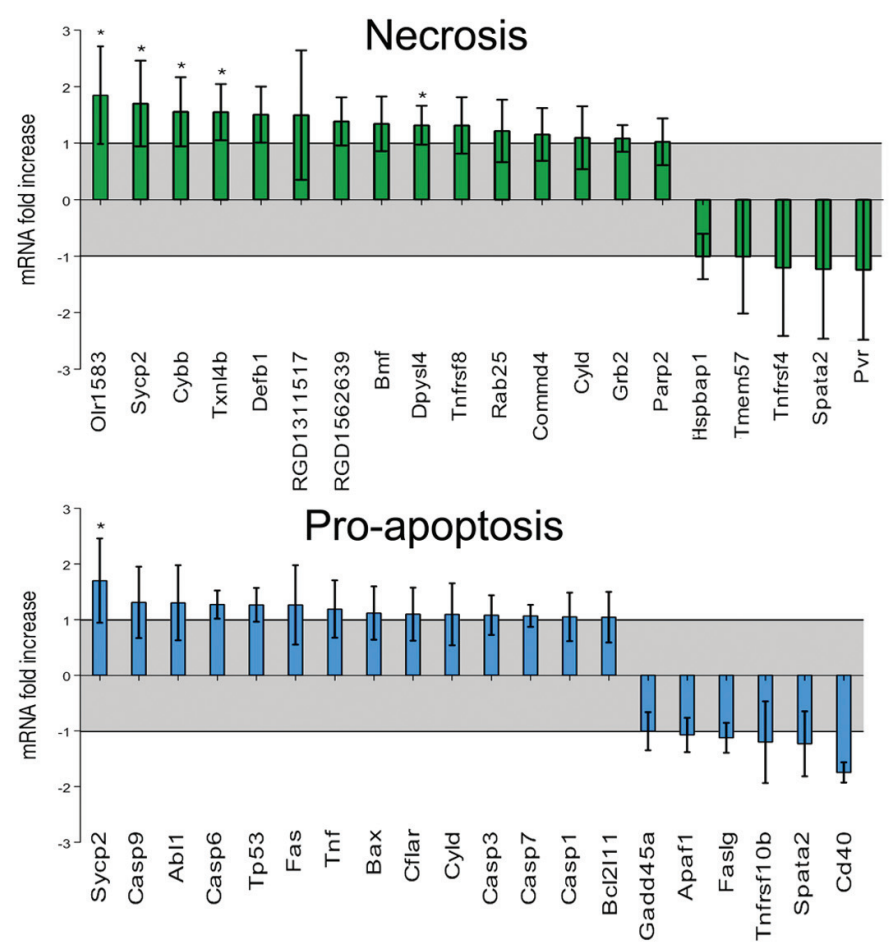

Anti-apoptosis
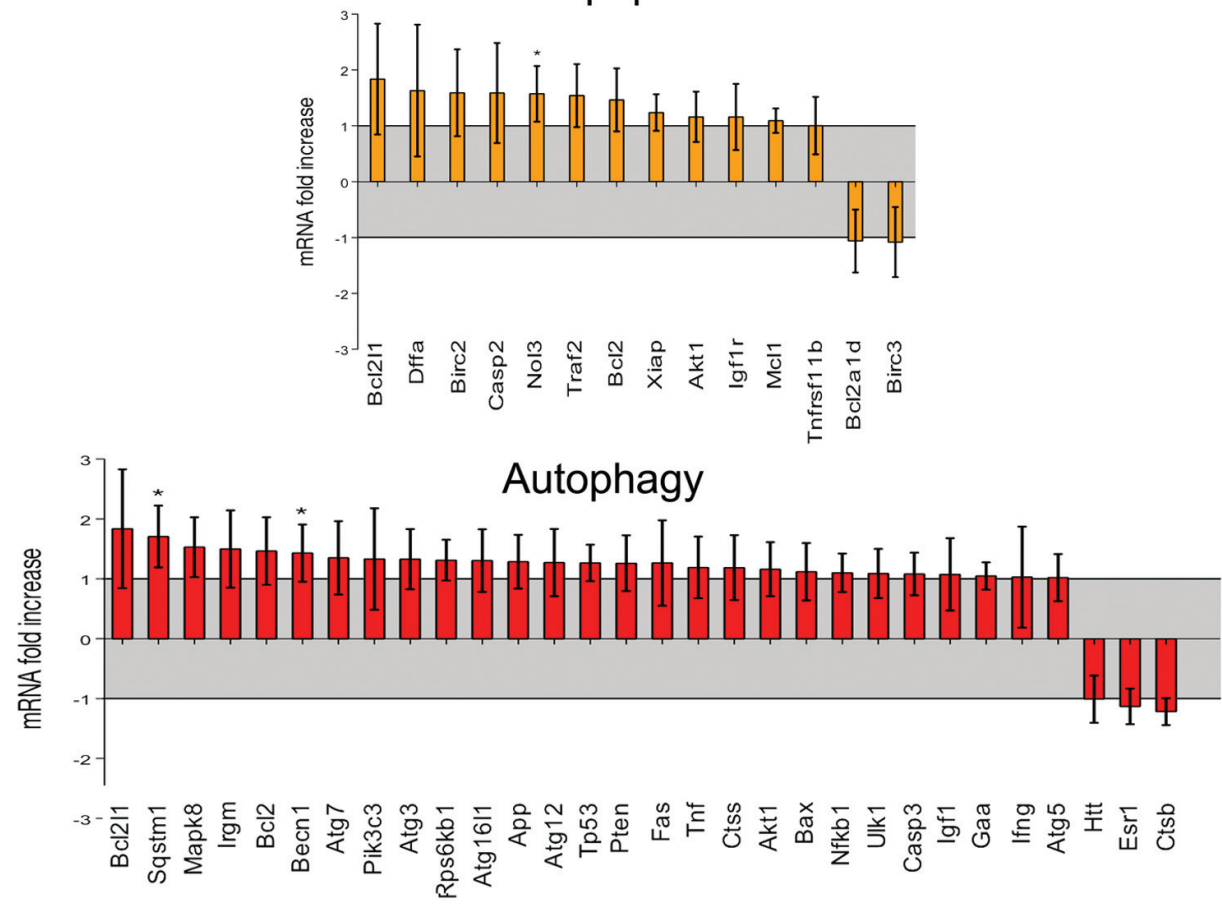

Figure 4. Regulation of genes after $15 \mathrm{~min}$ of helium postconditioning shown as fold increase or decrease in comparison to I/R15 per category. ${ }^{*} p<0.05$ the current study, in which histological analysis showed less cellular damage after He15 in comparison to I/R15. He15 slices exhibited less contraction band necrosis, edema, extravasation of red blood cells and granulocytes and fewer platelets/thrombi than I/R15 slices. Interestingly, histological slices of $30 \mathrm{~min}$ of helium showed excessive cellular damage in comparison to 15 min of helium. Again, this is in line with results from infarct size experiments analyzed by TTC staining, in which $30 \mathrm{~min}$ of helium during early reperfusion abrogated the protection that was seen after 15 min of HePOC (26). Prolonged episodes of helium inhalation did not protect the rabbit heart against I/R (27), neither did prolonged helium inhalation protect forearm endothelium in a study with male human volunteers (28).

From enzymatic and histologic assessment of cell damage after HePOC, we therefore drew the conclusion that (1) helium reduces cardiomyocyte damage which results in smaller infarct size, and (2) it does so only after 15 min of HePOC. This led to the investigation of transcriptional profiles of genes employed in cell death and survival pathways after $15 \mathrm{~min}$ of HePOC. Although mRNA expression profiles after $25 \mathrm{~min}$ of ischemia also were analyzed in this study, we focused on reperfusion because that seems to be the time window in which HePOC exerts its effects. Histological analysis showed a reduction of cell damage after HePOC at 15 min of reperfusion. From TTC staining, we know that the protective effect of 15 min of HePOC also can be found after $2 \mathrm{~h}$ of reperfusion $(15,21,26) ; 1 \mathrm{~h}$ and $45 \mathrm{~min}$ after the postconditioning stimulus is discontinued. It is very likely that within this time window both necrotic and apoptotic cell death are reduced by helium postconditioning, and that prosurvival mechanisms such as autophagy contribute substantially. We aimed to investigate whether signs of cellular survival on an mRNA expression level could be found as early as the $15 \mathrm{~min}$ reperfusion episode.

Separate studies investigating the effects of helium conditioning on specific proteins and their concomitant posttrans- 
Beclin-1

AAR

A

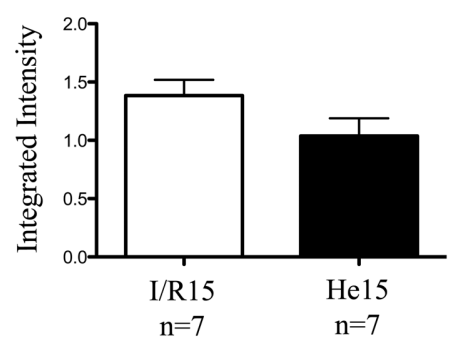

B

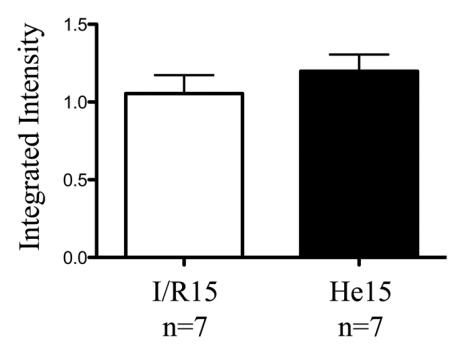

$\mathrm{C}$

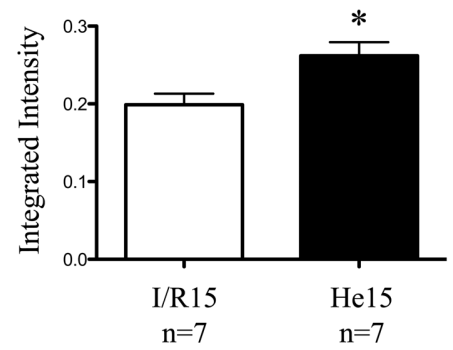

NAAR
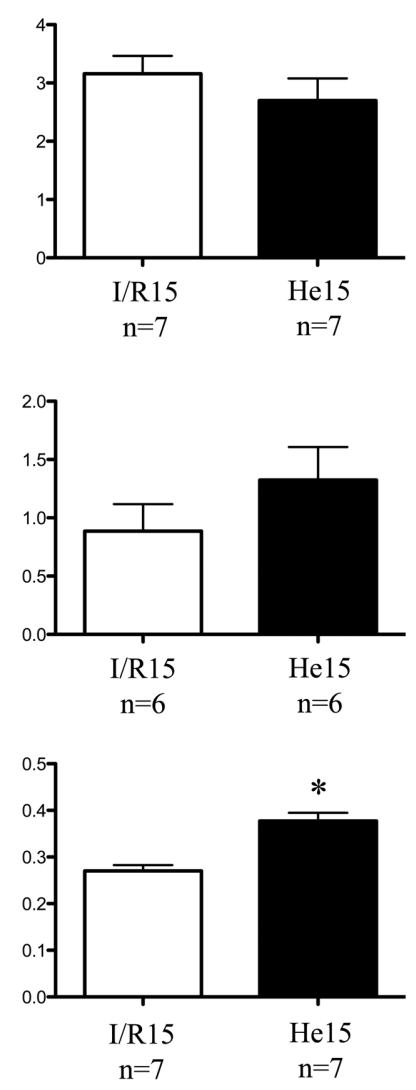

Figure 5. Protein levels of beclin-1. Data are shown as mean $\pm S E M,{ }^{*} P<0.05$. Ratios: (A) beclin-1:actin (cytosol fraction); (B) beclin-1:NaKATPase; (C) beclin-1:PHB1.

lational modifications in the cell death and survival pathways have been conducted (22). However, cell death and survival pathways interact, and various ways of cellular stress might trigger necrotic, apoptotic and autophagic pathways simultaneously, leading to activation of common downstream cell death elements or might offset each other (4). We therefore used PCR arrays to investigate four categories of cell death/survival simultaneously. Generally it is thought that necrosis occurs quickly and centrally, whereas apoptotic cell death takes a bit longer owing to the slowly orchestrated execution of the apoptotic cell death program and mainly occurs in the border zone of the area at risk (3). In this study, we investigated gene expression in the area at risk to find out which type of cell death is particularly affected by
HePOC. The trend of the current study suggests that orchestration of genes against apoptosis and proautophagy leads to the reduced cellular damage that is found in histological analyses at $15 \mathrm{~min}$ of reperfusion. This could make sense, as programmed cell death might play a far more important role than anticipated: in rats, chronic (7 d) ligation of a coronary artery resulted in a peak-mycocyte death within the first $4.5 \mathrm{~h}$ after ligation in which apoptosis predominated (29).

Autophagy is originally categorized as a survival mechanism in which cells consume their own proteins, lipids and organelles to maintain protein and organelle quality and to provide amino acids, energy and free fatty acids in case of nutrient deficiency. Cell constituents and parts of the cytoplasm are first engulfed in autophagosomes, after which fusion with lysosomes take place. Hereafter, degradation and recycling occurs. It is suggested that once this process becomes overactive, it becomes detrimental to cells and might end in autophagic cell death or in another type of cell death, such as apoptosis $(5,30,31)$. Yet, inhibition of autophagy might lead to cellular damage, for example due to an increased sensitivity to apoptotic signs, stressing the potential prosurvival role of autophagy (32). In vivo, sevoflurane late preconditioning (33) and ischemic POC (34) increased autophagic vacuoles and reduced infarct size.

In the current study, we found a simultaneous upregulation of genes employed in pathways against apoptosis and proautophagy; this could relate to the infarct size reduction that was observed after HePOC $(15,21,26)$ as well as the results from histological analysis in this study. Three significantly regulated genes Becn1 and Sqstm1 (autophagy) and Nol3 (antiapoptosis) in this analysis were of particular interest. Nol3 (nucleolar protein 3), which is also known as an apoptosis repressor with caspase recruitment domain (ARC), inhibits apoptosis on a level further down the apoptotic cascade as it directly binds to and inhibits caspase- 8 activity. Phosphorylation of ARC by protein kinase CK2 activates this protein, while calcineurin dephosphorylates and inactivates ARC (35). Not only did ARC overexpression decrease infarct size after I/R (5), anesthetic-induced preconditioning was associated with an increase of phosphorylation of ARC, a reduction in activity of calcineurin and a reduction in caspase- 8 activity and cytochrome c release (35). This is in line with our results; I/R decreases Nol3 expression while He15 increases it.

Our results show a general change in mRNA levels of genes involved in autophagy, including upregulation of the autophagy enhancer protein Becn1 after HePOC in comparison to I/R15. The inactivation of beclin-1 (Becn1), a protein involved in autophagosome formation, reduced infarct sizes (36) thereby suggesting a detrimental effect of au- 
tophagosome formation on infarct size. Becn1 loses its proautophagic function after interaction with $\mathrm{Bcl}-2$ (5), which is logical according to the hypothesis that an increased rate of autophagy in apoptotic cells probably leads to cell death (37). Thus, a combined downregulation of the antiapoptotic $\mathrm{BCl}-2$ with an upregulation of Becn1 during reperfusion facilitates cell death, presumably necrosis. In our study, I/R caused a downregulation of both Becn 1 and Bcl-2 in comparison to sham, which was diminished by helium postconditioning. Additionally, Becn1 protein levels were significantly higher after HePOC. These findings are comparable to results from a study in rats, in which ischemic postconditioning increased protein- and mRNA-levels of beclin-1 (34).

Protein analysis of Becn1 in cytosol, membrane and mitochondrial fractions of AAR and NAAR showed an identical pattern: after HePOC, there was a tendency toward a decrease in cytosol fractions, a tendency toward increase in the membrane fraction and a significant increase in the mitochondrial fraction. A possible reason for these findings is the translocation of the protein when autophagy is induced. The first step in autophagy is the formation of the phagophore; a membrane composed of different parts of organelles such as the Golgi complex, the ER and mitochondria. Subsequently, the autophagosome is formed and fuses with lysosomes, after which degradation of the cargo takes place $(38,39)$. Becn 1 is actively involved in the first step; it stimulates the formation of the phagophore and the autophagosome at the contact site of the ER and mitochondria (40). Hence, the increased Becn1 levels in the mitochondrial and membrane fractions. Becn 1 also is needed for autophagosome-lysosome fusion, a crucial step for an effective autophagy flux. Not only ischemic preconditioning, but also nonclassical preconditioning agents are known to induce autophagy and enhance the autophagic flux (41).

Albeit complex, autophagy flux can be measured in several ways. Sqstm1 (sequestosome 1) is generally considered a read out for autophagic activity; protein levels of Sqstm1 are regulated by autophagy as they are degraded in its machinery, due to its role in the delivery of ubiquitinated cargo to autophagosomes $(39,42,43)$. It is especially linked to mitophagy: the process in which depolarized or damaged mitochondria are directed toward degradation in the autophagosome. Discarding damaged constituents of mitochondria is essential in I/R injury and plays a role in aging and cardiovascular disease $(44,45)$. In mice, infarct size reduction after ischemic postconditioning was accompanied by translocation of Sqstm1 to mitochondria and presence of Parkin, an E3 ubiquitin ligase. In Parkin ${ }^{-/}$mice the translocation of Sqstm1 to mitochondria was absent and infarct size reduction was blunted (46). These findings are in line with our results, as the I/R-induced downregulation of Sqstm1 was attenuated after helium postconditioning. However, the mRNA upregulation after HePOC did not seem to translate to changes at a protein level. This could be explained by the fact that a true measurement of autophagic flux has not been done. From literature, it is known that upregulation of Sqstm mRNA does not always translate to an increase in protein levels (39). Sqstm1 protein levels are not only influenced by processing through the autophagosome machinery, they also are influenced by proteosomal degradation (39), a different pathway of protein degradation within a cell (43).

Although Figure 5 is particularly useful to observe trends, it also shows that some genes categorized in the necrotic pathway were upregulated significantly after He15: Olr1583, Sycp2, Cybb, Txnl4b and Dpysl4.

Their function in vivo is described in Supplementary Table S2. After a quick glance at these genes, it looks as if some unexpected genes play a role in cardioprotective mechanisms. Olr1583 and Dpysl4 are not directly known to be expressed in the heart, but do play a role in HePOC.

Olr1583 (Olfactory receptor 1583), a member of the olfactory gene family, is found predominantly in the olfactory epithelium of the nose and is involved in the recognition of specific odorants (47). However, evidence exists that at least one specific member of the olfactory receptor family also exists in the heart. Olfactory receptor 1 transcripts were detected in the developing heart, suggesting that the olfactory receptor might play a role in cardiac development (48). In the current study, we show that Olr1583 is expressed in the heart and that this expression is downregulated by I/R, but upregulated after He15. Dpysl4 (dihydropyrimidinase-like 4), also known as CRMP3, is expressed in the developing brain, but its function is unclear $(49,50)$. An inhibitory effect on brain development (50) as well as a crucial role in neurite outgrowth and axonal differentiation (49) has been described. We showed a downregulation of CRPM3 in heart tissue after I/ R in comparison to sham-operated animals and an attenuation of downregulation after He15.

Two other genes, Sycp2 and Txnl4b, are involved in the cell cycle and biological diversity. Sycp2 (synaptonemal complex protein 2) is part of the so-called synaptonemal complex, a meiosis-specific nuclear structure that is involved in recombination of chromosomes during the prophase, resulting in chromosomal crossover (51). Sycp2 therefore plays a role in genetic variation. Txnl4b (thioredoxinlike 4B) or DIM-2 is a gene required in cell cycle progression as it transits cells from the synthesis (S)-phase to the growth 2 (G2) phase, and it is involved in premRNA splicing (52). Pre-mRNA leads to different types of mRNA, which in turn results in different proteins. In a way, premRNA plays a role in the establishment of the vast diversity of proteins that exists in eukaryotes. I/R downregulated both Sycp 2 and Txnl4b, while He15 attenuated the downregulation of Sycp 2 and even upregulated $T x n l 4 b$.

Cybb (Cytochrome b-245, $\beta$ polypeptide), also known as Gp91-phox or Nox2, encodes for a protein called cytochrome b-245, which is a constituent of the NADPH-oxidase. The NADPH-oxidase produces superoxide and hydrogen peroxide in phagocytes that are used for the 
killing of pathogens (53) in vascular smooth muscle cells, endothelial cells $(54,55)$ and cardiomyocytes $(56)$. Nox2 is upregulated in infarcted areas after myocardial infarction in the rat (54) and after hypoxia-reoxygenation in porcine coronary artery endothelial cells (PCAEC) (55). Pharmacological or genetic blockade of the NAPDH oxidase in PCAECs reduced the hypoxia/reoxygenation-induced reactive oxygen species levels. Interestingly, this was accompanied by a reduction in vessel outgrowth (55), suggesting a role for NADPH oxidase in angiogenesis and neovascularization.

Nox2 and NADPH oxidase upregulation might be associated with cell death due to increased levels of oxidative stress, however, under certain circumstances, upregulation could signal prosurvival (57). It is not unlikely that the outcome depends on the intensity and extent of the ROS signal, the present kinases and caspases in the tissue, the stress signal that induced it and the type of tissue (57). Nox-induced ROS was shown to be involved in the differentiation of cardiomyocytes from embryonic stem cells and neonatal cardiomyocytes. Nox2 expression peaked at embryonic d 12, suggesting a critical role for Nox2 in early cardiomyogenesis. As Nox2 is also upregulated after myocardial infarction, it is not unlikely that it plays a role in differentiation of cardiac progenitor cells (56). In our study, I/R downregulated the Cybb/Nox2 gene, while He15 upregulated it. Taken together, the relative upregulation of Cybb/Nox2, Sycp2 and Txnl4b suggest that $\mathrm{HePOC}$ is possibly related to organ development and cell reproduction. This idea is underlined by the finding that Olr1583 and Dpysl4 are expressed in the heart and affected by HePOC.

A limitation of this study is the lack of subsequent experiments investigating protein levels of all the corresponding genes in the mRNA expression profile. Furthermore, posttranslational modifications often determine net function and the effect of a protein. Additionally, autophagy and mitophagy are complex processes, and their investigation requires sophisticated research techniques. However, the goal of this study was to obtain an insight in the cell death and survival pathways that are involved in HePOC.

Because of the aforementioned goal of this study, no experiments were conducted to obtain specific hemodynamic measurements such as pressure-volume loops. As no studies have been done to investigate the contractile properties of the myocardium after helium postconditioning, it would be interesting to use a conductance catheter in future experiments. However, calibrating the volume component is a complex procedure, as it can be done by ultrasound, blood conductivity or stroke volume measurements. This might limit its feasibility $(58,59)$. ECG recordings during I/R could point out another interesting problem often occurring in human coronary artery disease: (lethal) arrhythmia. In 1969, it was shown in dogs that heliox inhalation reduced the occurrence of ventricular fibrillation during the acute phase of coronary occlusion (60). It is questionable though, how relevant the problem of arrhythmia is in the small rodent model of regional I/R, as the dog and human heart differs in the presence of collateral arteries. Additionally, in humans, the presence of multiple atherosclerotic plaques (that is, multiple vessel disease) often poses the most significant threat. The likelihood of reducing I/R-induced arrhythmias by an intervention in a small rodent model is small when significant arrhythmia is not a dominant problem in the first place.

Our study does indicate that HePOC exhibits a wide array of effects on cell death and survival, and that it does so in an acute manner. A clue as to the underlying mechanism of HePOC could be found within the complex interplay of the abovementioned cellular processes. Beside the cell death and survival pathways, it is likely that oxidative stress pathways also are involved in helium postconditioning. Future research could focus on this direction, but should, in any case, comprise antiapoptotic and autophagic pathways as well as the long-term effect of HePOC on gene transcription and translation.

\section{CONCLUSION}

In conclusion, helium-induced cardioprotection by 15 min of POC seems to be associated with activation of prosurvival cell mechanisms. Helium influences the balance between pro- and antiapoptosis, in favor of genes directed against apoptosis. Simultaneously, it stimulates genes involved in autophagy and possibly cell reproduction and tissue development. This suggests that helium exerts its protective effects through a cell-surviving mechanism that comprises a whole set of pathways.

\section{DISCLOSURES}

The authors declare they have no competing interests as defined by Molecular Medicine, or other interests that might be perceived to influence the results and discussion reported in this paper.

\section{REFERENCES}

1. Kramarow E, Lubitz J, Francis R. (2013) Trends in the coronary heart disease risk profile of middleaged adults. Ann. Epidemiol. 23:31-4.

2. Ovize $\mathrm{M}$, et al. (2010) Postconditioning and protection from reperfusion injury: where do we stand? Position paper from the Working Group of Cellular Biology of the Heart of the European Society of Cardiology. Cardiovasc. Res. 87:406-23.

3. Hamacher-Brady A, Brady NR, Gottlieb RA. (2006) The interplay between pro-death and pro-survival signaling pathways in myocardial ischemia/reperfusion injury: apoptosis meets autophagy. Cardiovasc. Drugs Ther. 20:445-62.

4. Jain MV, et al. (2013) Interconnections between apoptotic, autophagic and necrotic pathways: implications for cancer therapy development. J. Cell. Mol. Med. 17:12-29.

5. Konstantinidis K, Whelan RS, Kitsis RN. (2012) Mechanisms of cell death in heart disease. Arterioscler. Thromb. Vasc. Biol. 32:1552-62.

6. Barach A. (1934) Use of helium as a new therapeutic gas. Proc. Soc. Exp. Bio. Med. 32:462-4.

7. Moraa I, Sturman N, McGuire T, van Driel ML. (2013) Heliox for croup in children. Cochrane Database Syst. Rev. 12:CD006822.

8. Rodrigo G, Pollack C, Rodrigo C, Rowe BH. (2006) Heliox for nonintubated acute asthma patients. Cochrane Database Syst. Rev. 18:CD002884.

9. Bennett MH, Lehm JP, Mitchell SJ, Wasiak J. (2012) Recompression and adjunctive therapy for decompression illness. Cochrane Database Syst Rev. 5:CD005277.

10. Oei GT, Weber NC, Hollmann MW, Preckel B. (2010) Cellular effects of helium in different organs. Anesthesiology. 112:1503-10. 
11. Huhn R, et al. (2012) Age-related loss of cardiac preconditioning: impact of protein kinase A. Exp. Gerontol. 47:116-21.

12. Pagel PS, Krolikowski JG. (2009) Transient metabolic alkalosis during early reperfusion abolishes helium preconditioning against myocardial infarction: restoration of cardioprotection by cyclosporin A in rabbits. Anesth. Analg. 108:1076-82.

13. Pagel PS, Krolikowski JG, Amour J, Warltier DC, Weihrauch D. (2009) Morphine reduces the threshold of helium preconditioning against myocardial infarction: the role of opioid receptors in rabbits. J. Cardiothorac. Vasc. Anesth. 23:619-24.

14. Pagel PS, et al. (2007) Noble gases without anesthetic properties protect myocardium against infarction by activating prosurvival signaling kinases and inhibiting mitochondrial permeability transition in vivo. Anesth. Analg. 105:562-9.

15. Huhn R, et al. (2009) Helium-induced early preconditioning and postconditioning are abolished in obese Zucker rats in vivo. J. Pharmacol. Exp. Ther. 329:600-7.

16. Heinen A, et al. (2008) Helium-induced preconditioning in young and old rat heart: impact of mitochondrial $\mathrm{Ca}(2+)$-sensitive potassium channel activation. Anesthesiology. 109:830-6.

17. Pagel PS, et al. (2008) Inhibition of glycogen synthase kinase or the apoptotic protein p53 lowers the threshold of helium cardioprotection in vivo: the role of mitochondrial permeability transition. Anesth. Analg. 107:769-75.

18. Pagel PS, et al. (2008) The mechanism of heliuminduced preconditioning: a direct role for nitric oxide in rabbits. Anesth. Analg. 107:762-8.

19. Pagel PS, et al. (2008) Reactive oxygen species and mitochondrial adenosine triphosphate-regulated potassium channels mediate helium-induced preconditioning against myocardial infarction in vivo. J. Cardiothorac. Vasc. Anesth. 22:554-9.

20. Huhn R, et al. (2009) Helium-induced late preconditioning in the rat heart in vivo. $\mathrm{Br}$. J. Anaesth. 102:614-19.

21. Oei GT, et al. (2012) Helium-induced cardioprotection of healthy and hypertensive rat myocardium in vivo. Eur. J. Pharmacol. 684:125-31.

22. Oei GT, Weber NC, Hollmann MW, Preckel B. (2010) Cellular effects of helium in different organs. Anesthesiology. 112:1503-10.

23. Institute of Laboratory Animal Resources; Commission on Life Sciences; National Research Council. (1996) Guide for the Care and Use of Laboratory Animals. Washington (DC): National Academy Press. See this URL for an electronic version: http:/ / www.nap.edu/catalog.php?record_id=5140

24. Ruijter JM, et al. (2009) Amplification efficiency: linking baseline and bias in the analysis of quantitative PCR data. Nucleic Acids Res. 37:e45

25. Battke F, Symons S, Nieselt K. (2010) Maydayintegrative analytics for expression data. $B M C$ Bioinformatics. 11:121.

26. Oei GT, Hollmann MW, Preckel B, Weber NC. (2012) Cardioprotection after a short episode of $70 \%$ helium inhalation at early reperfusion is abrogated by prolonged inhalation during reperfu- sion. Session presented at: 2012 American Society of Anesthesiologists annual meeting; 2013 Oct 13-17; Washington, DC. Abstract No. A151.

27. Hale SL, Vanderipe DR, Kloner RA. (2013) Continuous heliox breathing and the extent of anatomic zone of noreflow and necrosis following ischemia/reperfusion in the rabbit heart. Open Cardiovasc. Med. J. 8:1-5.

28. Lucchinetti E, et al. (2009) Helium breathing provides modest antiinflammatory, but no endothelial protection against ischemia-reperfusion injury in humans in vivo. Anesth. Analg. 109:101-8.

29. Kajstura J, et al. (1996) Apoptotic and necrotic myocyte cell deaths are independent contributing variables of infarct size in rats. Lab. Invest. 74:86-107.

30. Whelan RS, Kaplinskiy V, Kitsis RN. (2010) Cell death in the pathogenesis of heart disease: mechanisms and significance. Annu. Rev. Physiol. 72:19-44.

31. Gao L, et al. (2012) Inhibition of autophagy contributes to ischemic postconditioning-induced neuroprotection against focal cerebral ischemia in rats. PLoS One. 7:e46092.

32. Fimia GM, Piacentini M. (2010) Regulation of autophagy in mammals and its interplay with apoptosis. Cell. Mol. Life Sci. 67:1581-8.

33. Qiao S, et al. (2013) Delayed anesthetic preconditioning protects against myocardial infarction via activation of nuclear factor- $\mathrm{B}$ and upregulation of autophagy. J. Anesth. 27:251-60.

34. Wei C, Li H, Han L, Zhang L, Yang X. (2013) Activation of autophagy in ischemic postconditioning contributes to cardioprotective effects against ischemia/reperfusion injury in rat hearts. J. Cardiovasc. Pharmacol. 61:416-22.

35. Lu X, Moore PG, Liu H, Schaefer S. (2011) Phosphorylation of ARC is a critical element in the antiapoptotic effect of anesthetic preconditioning. Anesth. Analg. 112:525-31.

36. Matsui $Y$, et al. (2007) Distinct roles of autophagy in the heart during ischemia and reperfusion: roles of AMP-activated protein kinase and Beclin 1 in mediating autophagy. Circ. Res. 100:914-22.

37. Takagi H, Matsui Y, Sadoshima J. (2007) The role of autophagy in mediating cell survival and death during ischemia and reperfusion in the heart. Antioxid. Redox. Signal. 9:1373-81.

38. Kang R, Zeh HJ, Lotze MT, Tang D. (2011) The Beclin 1 network regulates autophagy and apoptosis. Cell Death Differ. 18:571-80.

39. Klionsky DJ, et al. (2012) Guidelines for the use and interpretation of assays for monitoring autophagy. Autophagy. 8:445-544.

40. Hamasaki M, et al. (2013) Autophagosomes form at ER-mitochondria contact sites. Nature. 495:389-93.

41. Gottlieb RA, Mentzer RM. (2010) Autophagy during cardiac stress: joys and frustrations of autophagy. Annu. Rev. Physiol. 72:45-59.

42. Komatsu M, Kageyama S, Ichimura Y. (2012) p62/SQSTM1/A170: physiology and pathology. Pharmacol. Res. 66:457-62.

43. Pankiv S, et al. (2007) p62/SQSTM1 binds directly to Atg8/LC3 to facilitate degradation of ubiquitinated protein aggregates by autophagy. J. Biol. Chem. 282:24131-45.
44. Wohlgemuth SE, Calvani R, Marzetti E. (2014) The interplay between autophagy and mitochondrial dysfunction in oxidative stress-induced cardiac aging and pathology. J. Mol. Cell. Cardiol. 71:62-70.

45. Redmann M, Dodson M, Boyer-Guittaut M, Darley-Usmar V, Zhang J. (2014) Mitophagy mechanisms and role in human diseases. Int. J. Biochem. Cell. Biol. 53C:127-33.

46. Huang C, et al. (2011) Preconditioning involves selective mitophagy mediated by Parkin and p62/SQSTM1. PLoS One. 6:e20975.

47. Malnic B, Godfrey PA, Buck LB. (2004) Correction for Malnic et al., The human olfactory receptor gene family, PNAS 2004 101:2584-2589. Proc. Natl. Acad. Sci. U. S. A. 101:7205.

48. Drutel G, et al. (1995) Cloning of OL1, a putative olfactory receptor and its expression in the developing rat heart. Receptors Channels. 3:33-40.

49. Quach TT, et al. (2008) CRMP3 is required for hippocampal CA1 dendritic organization and plasticity. FASEB J. 22:401-9.

50. Aylsworth A, Jiang SX, Desbois A, Hou ST. (2009) Characterization of the role of full-length CRMP3 and its calpain-cleaved product in inhibiting microtubule polymerization and neurite outgrowth. Exp. Cell Res. 315:2856-68.

51. Winkel K, Alsheimer M, Ollinger R, Benavente R. (2009) Protein SYCP2 provides a link between transverse filaments and lateral elements of mammalian synaptonemal complexes. Chromosoma. 118:259-67.

52. Simeoni F, Divita G. (2007) The Dim protein family: from structure to splicing. Cell. Mol. Life Sci. 64:2079-89.

53. Dworakowski R, Alom-Ruiz SP, Shah AM. (2008) NADPH oxidase-derived reactive oxygen species in the regulation of endothelial phenotype. Pharmacol. Rep. 60:21-8.

54. Fukui T, et al. (2001) Expression of p22-phox and gp91-phox, essential components of NADPH oxidase, increases after myocardial infarction. Biochem. Biophys. Res. Commun. 281:1200-6.

55. Chen JX, et al. (2007) NADPH oxidase modulates myocardial Akt, ERK1/2 activation, and angiogenesis after hypoxia-reoxygenation. Am. J. Physiol. Heart Circ. Physiol. 292:H1664-74.

56. Maejima Y, Kuroda J, Matsushima S, Ago T, Sadoshima J. (2011) Regulation of myocardial growth and death by NADPH oxidase. J. Mol. Cell. Cardiol. 50:408-16.

57. Bedard K, Krause KH. (2007) The NOX family of ROS-generating NADPH oxidases: physiology and pathophysiology. Physiol. Rev. 87:245-313.

58. Wei CL, et al. (2011) Does conductance catheter measurement system give consistent and reliable pressure-volume relations in rats? IEEE Trans. Biomed. Eng. 58:1804-13.

59. Pacher P, Nagayama T, Mukhopadhyay P, Bátkai S, Kass DA. (2008) Measurement of cardiac function using pressure-volume conductance catheter technique in mice and rats. Nat. Protoc. 3:1422-34.

60. Pifarré R, Cox WD, Jasuja M, Neville WE. (1969) Helium in the prevention of ventricular fibrillation. Dis. Chest. 56:135-8. 